\title{
EXAMPLES OF FRIEDRICHS MODEL OPERATORS WITH A CLUSTER POINT OF EIGENVALUES
}

\author{
SERGUEI I. IAKOVLEV
}

Received 26 February 2002

\begin{abstract}
A family of selfadjoint operators of the Friedrichs model is considered. These symmetric type operators have one singular point, zero of order $m$. For every $m>3 / 2$, we construct a rank 1 perturbation from the class Lip 1 such that the corresponding operator has a sequence of eigenvalues converging to zero. Thus, near the singular point, there is no singular spectrum finiteness condition in terms of a modulus of continuity of a perturbation for these operators in case of $m>3 / 2$.

2000 Mathematics Subject Classification: 47B06, 47B25.
\end{abstract}

1. Statement of the problem and main result. Consider selfadjoint operators $S_{m}, m>0$, given by

$$
S_{m}=\operatorname{sgn} t \cdot|t|^{m} \cdot+(\cdot, \varphi) \varphi
$$

on the domain of functions $u(t) \in L_{2}(\mathbb{R})$ such that $|t|^{m} u(t) \in L_{2}(\mathbb{R})$. Here $\varphi \in L_{2}(\mathbb{R})$ and $t$ is the independent variable. The action of the operator $S_{m}$ can be written as follows:

$$
\left(S_{m} u\right)(t)=\operatorname{sgn} t \cdot|t|^{m} u(t)+\varphi(t) \int_{\mathbb{R}} u(x) \overline{\varphi(x)} d x .
$$

The function $\varphi$ is assumed to satisfy the smoothness condition

$$
|\varphi(t+h)-\varphi(t)| \leq \omega(|h|), \quad|h| \leq 1,
$$

where the function $\omega(t)$ (the modulus of continuity of the function $\varphi$ ) is monotone and satisfies a Dini condition

$$
\omega(t) \downarrow 0 \quad \text { as } t \downarrow 0, \quad \int_{0}^{1} \frac{\omega(t)}{t} d t<\infty .
$$

For the operators $S_{m}$, the absolutely continuous spectrum fills the real axis $\mathbb{R}$. The behavior of the singular spectrum of the operators $S_{m}$ is of interest. Note that we define the singular spectrum as the union of the point spectrum and the singular continuous one. The structure of the spectrum $\sigma_{\text {sing }}\left(S_{1}\right)$ (the singular spectrum of the operator $S_{1}=t \cdot+(\cdot, \varphi) \varphi$ ) has been studied in detail in $[1,2,3,4,5,6,7,8,9]$. It is shown in $[1,7]$ that for this operator, 
there exists an exact condition of the singular spectrum finiteness. Namely, if $\omega(t)=O(\sqrt{t})$ as $t \rightarrow 0^{+}, \sigma_{\text {sing }}\left(S_{1}\right)$ consists of at most a finite number of eigenvalues of finite multiplicity (the singular continuous spectrum is missing). But if liminf $\omega(t) / \sqrt{t}=+\infty$ as $t \rightarrow 0^{+}$, then we construct examples showing that a nontrivial singular spectrum appears, in particular, the operator $S_{1}$ has accumulation points of eigenvalues. Note that the real appearance of a nontrivial singular spectrum in the Friedrichs model was, for the first time, shown by Pavlov and Petras [8].

Using the simple change of variables $\operatorname{sgn} t \cdot|t|^{m}=x$, we can show that outside any neighborhood of the origin, the structure of the spectrum $\sigma_{\text {sing }}\left(S_{m}\right)$ is identical with the one of the operator $S_{1}$. This is due to the smoothness of this change of variables outside any neighborhood of the origin, and also to the local character of the main results of [1, 2, 3, 4, 5, 6, 7, 8, 9]. Namely, suppose that conditions (1.3), (1.4), and also some additional conditions on the function $\varphi$ are fulfilled only in a certain interval $(c, d) \subset \mathbb{R}$, then, the main results of $[1,2,3,4,5,6,7,8,9]$, concerning the structure of $\sigma_{\text {sing }}\left(S_{1}\right)$, will remain true in any closed subinterval $\Delta \subset(c, d)$. At the same time, as shown in this paper, for the operator $S_{m}, m>3 / 2$, the behavior of the singular spectrum has quite different character in a neighborhood of the origin. In this case, it turns out that the singular spectrum can appear for any modulus of continuity $\omega(t)$. Hence, near zero, there is no condition of the singular spectrum finiteness in terms of the modulus of continuity of $\varphi(t)$ like for the operator $S_{1}$. Here, we can also use the pointed change of variables but, since, for instance, $\left(\operatorname{sgn} t \cdot|t|^{m}\right)_{\left.\right|_{t=0}}^{\prime}=0$ for $m>1$, it is not smooth (that is, not a diffeomorphism) near zero. In this sense, zero is a singular point of the operators $S_{m}, m \neq 1$, and needs a special attention.

We start with a formulation of the main theorem of this paper on the construction of a function $\varphi(t)$ such that the corresponding operator $S_{m}, m>3 / 2$, has a nontrivial singular spectrum near the singular point zero and, in particular, a sequence of eigenvalues converging to the origin. The proof of this theorem will be obtained as a combination of a sequence of lemmas. Observe that the actual modulus of continuity $\widetilde{\omega}(h):=\sup \left\{\left|\varphi\left(t_{1}\right)-\varphi\left(t_{2}\right)\right|:\left|t_{1}-t_{2}\right|<h\right\}$ of the function $\varphi$ always satisfies the additional constraint of semiadditivity $\widetilde{\omega}\left(t_{1}+t_{2}\right) \leq \widetilde{\omega}\left(t_{1}\right)+\widetilde{\omega}\left(t_{2}\right)$ for all $t_{1}, t_{2} \geq 0$.

THEOREM 1.1 (main result). Let a nonnegative, monotone function $\omega(t)$, $t \geq 0$, be semiadditive such that $\omega\left(t_{1}+t_{2}\right) \leq \omega\left(t_{1}\right)+\omega\left(t_{2}\right)$ for all $t_{1}, t_{2} \geq 0$. Then for any $m>3 / 2$, a compactly supported function $\varphi$ satisfying the smoothness condition $|\varphi(t+h)-\varphi(t)| \leq \omega(|h|), h \in \mathbb{R}$, is constructed and such that the corresponding operator $S_{m}=\operatorname{sgn} t \cdot|t|^{m} \cdot+(\cdot, \varphi) \varphi$ has a sequence of eigenvalues converging to zero.

Note that the result of Theorem 1.1 can be formulated in terms of real zeros of some analytic functions. Define in the upper half plane an analytic function 
$M_{m}(z)$ in the following way:

$$
M_{m}(z)=1+\int_{-\infty}^{+\infty} \frac{\left|\varphi^{2}(t)\right|}{\operatorname{sgn} t \cdot|t|^{m}-z} d t, \quad \operatorname{Im} z>0 .
$$

It is easily shown that under conditions (1.3) and (1.4), the function $M_{m}(z)$ is continuously extended up to the real axis on the intervals $(-\infty ; 0)$ and $(0 ;+\infty)$. We define, for $\lambda \in \mathbb{R} \backslash\{0\}$, the value $M_{m}(\lambda):=M_{m}(\lambda+i 0)$ and the roots set $N:=$ $\left\{\lambda \in \mathbb{R} \backslash\{0\}: M_{m}(\lambda)=0\right\}$. Then, we have the following inclusion $\sigma_{\text {sing }}\left(S_{m}\right) \subseteq$ $N \cup\{0\}$. (See, e.g., [10] where similar assertions are proved for the function $1+\int_{-\infty}^{+\infty}\left(\left|\varphi^{2}(t)\right| /\left(t^{2}-z\right)\right) d t$ or [8] for the function $1+\int_{-\infty}^{+\infty}\left(\left|\varphi^{2}(t)\right| /(t-z)\right) d t$.) Further, the exact condition $\omega(t)=O(\sqrt{t})$, as $t \rightarrow 0^{+}$, appears to guarantee that outside any neighborhood of the origin, there is at most a finite number of zeros of the function $M_{m}(\lambda)$. At the same time, Theorem 1.1 means that, for $m>3 / 2$, the function $M_{m}(\lambda)$ can have a sequence of zeros converging to the origin for any monotone, nonnegative, and semiadditive function $\omega$ satisfying condition (1.4).

\section{Construction of the function $\varphi$}

LEMmA 2.1. Let the function $\varphi$ belong to $L_{2}(\mathbb{R})$. Then a point $\lambda \in \mathbb{R}$ is an eigenvalue of the operator $S_{m}=\operatorname{sgn} t \cdot|t|^{m} \cdot+(\cdot, \varphi) \varphi$ if and only if the following conditions hold:

$$
\begin{gathered}
\frac{\varphi(t)}{\operatorname{sgn} t \cdot|t|^{m}-\lambda} \in L_{2}(\mathbb{R}), \\
1+\int_{-\infty}^{+\infty} \frac{\left|\varphi^{2}(t)\right|}{\operatorname{sgn} t \cdot|t|^{m}-\lambda} d t=0 .
\end{gathered}
$$

Proof. Solving the eigenvalue problem $S_{m} v=\lambda v$, we find that $v(t)=$ $C \varphi(t) /\left(\operatorname{sgn} t \cdot|t|^{m}-\lambda\right)$. (Remark that from now on, we denote by $C$ various constants which may be different even in a single chain of inequalities.) This expression will be an eigenfunction if it is from the space $L_{2}(\mathbb{R})$ and satisfies the equation $S_{m} v-\lambda v=0$, that is,

$$
\left(\operatorname{sgn} t \cdot|t|^{m}-\lambda\right) \frac{C \varphi(t)}{\operatorname{sgn} t \cdot|t|^{m}-\lambda}+\varphi(t) \int_{-\infty}^{+\infty} \frac{C\left|\varphi^{2}(x)\right|}{\operatorname{sgn} x \cdot|x|^{m}-\lambda} d x=0
$$

or

$$
\left(1+\int_{-\infty}^{+\infty} \frac{\left|\varphi^{2}(x)\right|}{\operatorname{sgn} x \cdot|x|^{m}-\lambda} d x\right) \varphi(t)=0
$$

The proof is complete. 
LEMMA 2.2. Suppose that a nonnegative, monotone function $\omega(t)$ is semiadditive $\omega\left(t_{1}+t_{2}\right) \leq \omega\left(t_{1}\right)+\omega\left(t_{2}\right)$ for all $t_{1}, t_{2} \geq 0$. If $\liminf _{t \rightarrow 0^{+}} \omega(t) / t=0$, then $\omega(t)=0$ for all $t \geq 0$.

Proof. According to the semiadditivity, $\omega(n t) \leq n \omega(t), n \in \mathbb{N}$. Therefore, using the notation $[t]$ for the integer part of t, by the monotonicity of $\omega(t)$, we have for all $t, x>0$,

$$
\begin{aligned}
\omega(t) & =\omega\left(\left(\frac{t}{x}\right) x\right) \leq \omega\left(\left(\left[\frac{t}{x}\right]+1\right) x\right) \leq\left(\left[\frac{t}{x}\right]+1\right) \omega(x) \\
& \leq\left(\frac{t}{x}+1\right) \omega(x)=(t+x) \frac{\omega(x)}{x} .
\end{aligned}
$$

Thus,

$$
\begin{aligned}
\omega(t) & \leq \liminf _{x \rightarrow 0^{+}}(t+x) \frac{\omega(x)}{x}=\lim _{\delta \rightarrow 0^{+}} \inf _{0<x<\delta}(t+x) \frac{\omega(x)}{x} \\
& \leq \lim _{\delta \rightarrow 0^{+}}(t+\delta) \inf _{0<x<\delta} \frac{\omega(x)}{x}=t \cdot \liminf _{x \rightarrow 0^{+}} \frac{\omega(x)}{x}=0 .
\end{aligned}
$$

The lemma is proved.

COROLLARY 2.3. If additionally $\omega(t) \rightarrow 0^{+}$as $t \rightarrow 0^{+}$, then for any $t_{0}>0$, there exists a constant $C>0$ such that

$$
C t \leq \omega(t), \quad t \in\left[0 ; t_{0}\right]
$$

Proof. It suffices to show that there exists a positive $\varepsilon$ less than $t_{0}$ such that the inequality $C t \leq \omega(t)$ is fulfilled with a certain constant $C>0$ for all $t \in[0 ; \varepsilon]$. If this is the case, put $\tilde{C}:=\min \left\{C, \omega(\varepsilon) / t_{0}\right\}$. Then, the inequality $\tilde{C} t \leq \omega(t)$ already holds for all $t \in\left[0 ; t_{0}\right]$. Indeed, using the monotonicity of $\omega$, we have for $t \in\left[\varepsilon ; t_{0}\right]$

$$
\tilde{C} t \leq \frac{\omega(\varepsilon)}{t_{0}} \cdot t \leq \inf _{x \in\left[\varepsilon ; t_{0}\right]}\left(\frac{\omega(x)}{x}\right) \cdot t \leq \frac{\omega(t)}{t} \cdot t=\omega(t) .
$$

We prove that there are such constants $\varepsilon$ and $C$. By assuming the converse, we construct two positive sequences $C_{k}$ and $t_{k}$ tending to 0 such that $\omega\left(t_{k}\right) \leq C_{k} t_{k}$. Hence, $\lim _{k \rightarrow+\infty} \omega\left(t_{k}\right) / t_{k}=0$. Since

$$
\liminf _{t \rightarrow 0^{+}} \frac{\omega(t)}{t} \leq \liminf _{k \rightarrow+\infty} \frac{\omega\left(t_{k}\right)}{t_{k}}=0
$$

by Lemma 2.2, $\omega=0$. This completes the proof.

Remark that the nonnegativity of $\omega$ is a consequence of its semiadditivity and monotonicity as $\omega(t+0) \leq \omega(t)+\omega(0)$, and hence $0 \leq \omega(0) \leq \omega(t)$. 
Corollary 2.3 shows that it is sufficient to prove Theorem 1.1 for $\omega(t)=C_{\omega} t$ with an arbitrary constant $C_{\omega}>0$.

Let $\left\{u_{n}\right\}_{n=0}^{+\infty}$ and $\left\{\varepsilon_{n}\right\}_{n=1}^{+\infty}$ be two sequences from the interval $\left(0 ; 10^{-1}\right)$ satisfying the condition

$$
u_{n}<\varepsilon_{n}<\frac{u_{n-1}}{8}, \quad n=1,2, \ldots
$$

On the real axis, we define a sequence of functions $\varphi_{n}$ as follows:

$$
\varphi_{n}(t):= \begin{cases}\omega\left(t-\frac{u_{n}}{2}\right), & t \in\left[\frac{1}{2} u_{n} ; \frac{3}{4} u_{n}\right], \\ \omega\left(u_{n}-t\right), & t \in\left[\frac{3}{4} u_{n} ; u_{n}\right], \\ 0, & t \notin\left[\frac{1}{2} u_{n} ; u_{n}\right],\end{cases}
$$

where $\omega(t)=C_{\omega} t$.

We will show that for any real-valued Lipschitz function $\gamma(t)$ compactly supported in the interval $(-\infty ;-1)$, it is possible to select the sequences $u_{n}$ and $\varepsilon_{n}$, and a bounded sequence of nonnegative numbers $\left\{c^{n}\right\}_{n=1}^{+\infty}$ such that the points $\lambda_{n}:=\left(u_{n}+\varepsilon_{n}\right)^{m}$ will be eigenvalues of the operator $S_{m}=\operatorname{sgn} t$. $|t|^{m} \cdot+(\cdot, \varphi) \varphi$ with the function

$$
\varphi(t):=K \cdot \sum_{k=1}^{+\infty}\left(c^{k}\right)^{1 / 2} \varphi_{k}(t)+\gamma(t) .
$$

Here $K>0$ is a parameter. It will be shown that for all $K$ large enough, the function $\varphi$ satisfies the required smoothness condition $|\varphi(t+h)-\varphi(t)| \leq$ $\omega(|h|), t, h \in \mathbb{R}$.

LEMMA 2.4. For the points $\lambda_{n}$ to be eigenvalues of the operator $S_{m}$ defined by (1.1), it is necessary and sufficient that

$$
\begin{gathered}
\int_{-\infty}^{+\infty} \frac{\left|\varphi^{2}(t)\right|}{\operatorname{sgn} t \cdot|t|^{m}-\lambda_{1}} d t=-1, \\
\int_{-\infty}^{+\infty} \frac{\left|\varphi^{2}(t)\right|}{\left(\operatorname{sgn} t \cdot|t|^{m}-\lambda_{n}\right)\left(\operatorname{sgn} t \cdot|t|^{m}-\lambda_{n+1}\right)} d t=0, \quad n=1,2, \ldots .
\end{gathered}
$$

Proof. We need to verify conditions (2.1) and (2.2) for $\lambda=\lambda_{n}, n=1,2, \ldots$. As $u_{n}<u_{n-1} / 8$, the supports of the functions $\varphi_{n}$ are disjoint. Thus, the function $\varphi$ is bounded and compactly supported. According to (2.10), $\lambda_{n}=$ $\left(u_{n}+\varepsilon_{n}\right)^{m}<t^{m}$ for $t \geq u_{n-1} / 2$. Therefore, $\varphi$ vanishes identically in a neighborhood of $\lambda_{n}$. Hence, condition (2.1) is fulfilled for any $\lambda=\lambda_{n}, n=1,2, \ldots$. 
Now putting in (2.2) $\lambda=\lambda_{n}, n=1,2, \ldots$ we then pass from the obtained system of equations to an equivalent one by subtracting the $n$th equation from the $(n+1)$ st for $n=1,2, \ldots$. As a result, we get system (2.13) and (2.14). The lemma is proved.

Since $\varphi(t)=0$ for $t>\lambda_{1}$, it follows that

$$
\alpha_{m}:=\int_{-\infty}^{+\infty} \frac{\varphi^{2}(t)}{\operatorname{sgn} t \cdot|t|^{m}-\lambda_{1}} d t<0
$$

Therefore, after solving the homogeneous system (2.14), the first equality (2.13) will be satisfied by replacing the function $\varphi$ by $\varphi / \sqrt{\left|\alpha_{m}\right|}$.

Substituting expression (2.12) for $\varphi(t)$ in (2.14), we obtain a system of linear equations for the unknowns $c^{n}$,

$$
\sum_{k=1}^{n-1}\left(-d_{n k} c^{k}\right)+d_{n n} c^{n}+\sum_{k=n+1}^{+\infty}\left(-d_{n k} c^{k}\right)=\gamma_{n}, \quad n=1,2, \ldots,
$$

with the coefficients

$$
\begin{aligned}
d_{n k} & :=K^{2} \int_{u_{k} / 2}^{u_{k}} \frac{\varphi_{k}^{2}(t)}{\left|\left(t^{m}-\lambda_{n}\right)\left(t^{m}-\lambda_{n+1}\right)\right|} d t, \\
\gamma_{n} & :=\int_{-\infty}^{-1} \frac{\gamma^{2}(t)}{\left(|t|^{m}+\lambda_{n}\right)\left(|t|^{m}+\lambda_{n+1}\right)} d t .
\end{aligned}
$$

In the next section we show that the linear system (2.16) has a nonnegative solution in the space $l_{\infty}$ of bounded sequences.

\section{Solution of the linear system}

LEMmA 3.1. The coefficients $d_{n k}$ of the linear system (2.16) satisfy the following inequalities:

$$
\begin{gathered}
d_{n n} \geq K^{2} \frac{C_{1}}{\varepsilon_{n}^{m} u_{n}^{m-3}}, \\
\sum_{k=1}^{n-1} d_{n k} \leq K^{2} \frac{C_{2}}{u_{n-1}^{2 m}}, \\
\sum_{k=n+1}^{+\infty} d_{n k} \leq K^{2} \frac{C_{3} u_{n+1}^{3}}{\varepsilon_{n}^{m} \varepsilon_{n+1}^{m}},
\end{gathered}
$$

with some positive constants $C_{1}, C_{2}$, and $C_{3}$.

PRoof. For arbitrary $a, b>0$ and $m \geq 1$, the following inequality obviously holds:

$$
|a-b| \cdot \max \left\{a^{m-1}, b^{m-1}\right\} \leq\left|a^{m}-b^{m}\right| \leq|a-b| \cdot \max \left\{a^{m-1}, b^{m-1}\right\} m \text {. (3.4) }
$$


Thus,

$$
\begin{aligned}
\frac{1}{|a-b| \cdot \max \left\{a^{m-1}, b^{m-1}\right\} m} & \leq \frac{1}{\left|a^{m}-b^{m}\right|} \\
& \leq \frac{1}{|a-b| \cdot \max \left\{a^{m-1}, b^{m-1}\right\}} .
\end{aligned}
$$

Using inequalities (3.5) and (2.10) with elementary estimates and a simple change of variables $x:=u_{n}-t$, we get

$$
\begin{aligned}
d_{n n} \geq & K^{2} \int_{3 u_{n} / 4}^{u_{n}} \frac{\omega^{2}\left(u_{n}-t\right) d t}{\left(\left(u_{n}+\varepsilon_{n}\right)^{m}-t^{m}\right) \cdot\left(t^{m}-\left(u_{n+1}+\varepsilon_{n+1}\right)^{m}\right)} \\
\geq & K^{2} \int_{3 u_{n} / 4}^{u_{n}} \omega^{2}\left(u_{n}-t\right) d t \\
& \cdot \frac{1}{\left(\left(u_{n}+\varepsilon_{n}\right)^{m}-\left(3 u_{n} / 4\right)^{m}\right) \cdot\left(u_{n}^{m}-\left(u_{n+1}+\varepsilon_{n+1}\right)^{m}\right)} \\
\geq & K^{2} \int_{0}^{u_{n} / 4} \omega^{2}(x) d x \\
& \cdot \frac{1}{\left(m\left(u_{n}+\varepsilon_{n}-(3 / 4) u_{n}\right)\left(u_{n}+\varepsilon_{n}\right)^{m-1}\right) \cdot\left(m\left(u_{n}-u_{n+1}-\varepsilon_{n+1}\right) u_{n}^{m-1}\right)} \\
\geq & K^{2} \frac{C_{\omega}^{2}}{3}\left(\frac{u_{n}}{4}\right)^{3} \cdot \frac{1}{\left(m\left(u_{n} / 4+\varepsilon_{n}\right)\left(u_{n}+\varepsilon_{n}\right)^{m-1}\right) \cdot\left(m u_{n}^{m}\right)} \\
\geq & K^{2} C \frac{u_{n}^{3}}{\left(u_{n}+\varepsilon_{n}\right)^{m} \cdot u_{n}^{m}} \geq K^{2} \frac{C_{1}}{\varepsilon_{n}^{m} \cdot u_{n}^{m-3}} .
\end{aligned}
$$

Inequality (3.2) is established analogously,

$$
\begin{aligned}
\sum_{k=1}^{n-1} d_{n k}= & \sum_{k=1}^{n-1} K^{2} \int_{u_{k} / 2}^{u_{k}} \frac{\varphi_{k}^{2}(t) d t}{\left(t^{m}-\left(u_{n}+\varepsilon_{n}\right)^{m}\right) \cdot\left(t^{m}-\left(u_{n+1}+\varepsilon_{n+1}\right)^{m}\right)} \\
\leq & K^{2} \sum_{k=1}^{n-1} \int_{u_{k} / 2}^{u_{k}} \frac{\varphi_{k}^{2}(t) d t}{\left(\left(u_{n-1} / 2\right)^{m}-\left(u_{n}+\varepsilon_{n}\right)^{m}\right) \cdot\left(\left(u_{n-1} / 2\right)^{m}-\left(u_{n+1}+\varepsilon_{n+1}\right)^{m}\right)} \\
\leq & K^{2} \int_{0}^{u_{1}}\left(\sum_{k=1}^{+\infty} \varphi_{k}^{2}(t)\right) d t \frac{1}{\left(\left(u_{n-1} / 2-u_{n}-\varepsilon_{n}\right)\left(u_{n-1} / 2\right)^{m-1}\right)} \\
& \times \frac{1}{\left(\left(u_{n-1} / 2-u_{n+1}-\varepsilon_{n+1}\right)\left(u_{n-1} / 2\right)^{m-1}\right)} \\
\leq & K^{2} C \frac{1}{\left(u_{n-1} / 2-u_{n-1} / 8-u_{n-1} / 8\right)^{2} \cdot\left(u_{n-1} / 2\right)^{2(m-1)}} \leq K^{2} \frac{C_{2}}{u_{n-1}^{2 m}} .
\end{aligned}
$$


We took into account that the functions $\varphi_{k}(t)$ are uniformly bounded and have disjoint supports, therefore $\int_{0}^{u_{1}}\left(\sum_{k=1}^{+\infty} \varphi_{k}^{2}(t)\right) d t \leq C$. Finally, we have

$$
\begin{aligned}
& \sum_{k=n+1}^{+\infty} d_{n k} \\
& =K^{2} \sum_{k=n+1}^{+\infty} \int_{u_{k} / 2}^{u_{k}} \frac{\varphi_{k}^{2}(t) d t}{\left(\left(u_{n}+\varepsilon_{n}\right)^{m}-t^{m}\right) \cdot\left(\left(u_{n+1}+\varepsilon_{n+1}\right)^{m}-t^{m}\right)} \\
& \leq K^{2} \sum_{k=n+1}^{+\infty} \int_{u_{k} / 2}^{u_{k}} \frac{\varphi_{k}^{2}(t) d t}{\left(\left(u_{n}+\varepsilon_{n}\right)^{m}-u_{n+1}^{m}\right) \cdot\left(\left(u_{n+1}+\varepsilon_{n+1}\right)^{m}-u_{n+1}^{m}\right)} \\
& \leq K^{2} \sum_{k=n+1}^{+\infty} \int_{u_{k} / 2}^{u_{k}} \frac{\omega^{2}\left(u_{k}-t\right) d t}{\left(\left(u_{n}+\varepsilon_{n}-u_{n+1}\right)\left(u_{n}+\varepsilon_{n}\right)^{m-1}\right) \cdot\left(\varepsilon_{n+1}\left(u_{n+1}+\varepsilon_{n+1}\right)^{m-1}\right)} \\
& \leq K^{2} \sum_{k=n+1}^{+\infty} \int_{u_{k} / 2}^{u_{k}} \frac{\omega^{2}\left(u_{n+1}-t\right) d t}{\left(\left(u_{n}+\varepsilon_{n}-u_{n} / 8\right)\left(u_{n}+\varepsilon_{n}\right)^{m-1}\right) \cdot \varepsilon_{n+1}\left(u_{n+1}+\varepsilon_{n+1}\right)^{m-1}} \\
& \leq K^{2} \int_{0}^{u_{n+1}} \omega^{2}\left(u_{n+1}-t\right) d t \frac{C}{\left(u_{n}+\varepsilon_{n}\right)^{m} \cdot \varepsilon_{n+1}\left(u_{n+1}+\varepsilon_{n+1}\right)^{m-1}} \\
& \leq K^{2} u_{n+1}^{3} \frac{C_{3}}{\varepsilon_{n}^{m} \cdot \varepsilon_{n+1}^{m}} \text {. }
\end{aligned}
$$

The lemma is proved.

We rewrite system (2.16) in matrix form

$$
(I+A) \vec{c}=f,
$$

where the column vectors $\vec{c}=\left(c^{1}, c^{2}, \ldots\right)^{T}, f=\left(\gamma_{1} / d_{11}, \gamma_{2} / d_{22}, \ldots\right)^{T}$, and the infinite matrix $A$ has the entries $(A)_{n k}=\left(\delta_{n k}-1\right) \cdot d_{n k} / d_{n n}$. Equation (3.9) will be considered in the Banach space $l_{\infty}$.

In the sequel, we consider the sequences $u_{n}$ and $\varepsilon_{n}$ defined as follows:

$$
u_{n}=u_{n-1}^{\alpha}, \quad \varepsilon_{n}=u_{n-1}^{\beta}, \quad n=1,2, \ldots
$$

with some $u_{0} \in\left(0 ; 10^{-1}\right)$ and $\alpha>\beta>2$. It is evident that inequality (2.10) is fulfilled.

LEMMA 3.2. For every $m>3 / 2$, the numbers $\alpha$ and $\beta$ can be found satisfying the inequality $\alpha>\beta>2$ such that the following estimate:

$$
d_{n n} \geq K^{2} C_{1}
$$

holds for all $n=1,2, \ldots$. 
Proof. Substituting (3.10) in (3.1), we get

$$
d_{n n} \geq K^{2} \frac{C_{1}}{u_{n-1}^{\beta m+\alpha(m-3)}}
$$

As $u_{n-1} \in(0 ; 1)$ for $m \geq 3$, the assertion of the lemma is true with any $\alpha>\beta>$ 2 . Now let $m \in(3 / 2 ; 3)$. Then, the inequality $\beta m+\alpha(m-3)>0$ is fulfilled if $\alpha<\beta m /(3-m)$. It must be $\alpha>\beta>2$, therefore we require that $\beta m /(3-m)>$ $\beta$. As the last inequality holds for any $\beta>0$, we can take for $m \in(3 / 2 ; 3)$ any

$$
\beta>2, \quad \alpha \in\left(\beta ; \frac{\beta m}{(3-m)}\right)
$$

This completes the proof.

By (2.18), $\gamma_{n}>0$, and

$$
\gamma_{n}=\int_{-\infty}^{-1} \frac{|\gamma(t)|^{2} d t}{\left(|t|^{m}+\lambda_{n}\right)\left(|t|^{m}+\lambda_{n+1}\right)} \leq \int_{-\infty}^{-1}|\gamma(t)|^{2} d t \equiv\|\gamma\|_{2}^{2} .
$$

Hence, for the $l_{\infty}$-norm of the vector $f$ equal to $\|f\|_{\infty}=\sup _{n}\left(\gamma_{n} / d_{n n}\right)$, we obtain the following estimate:

$$
\|f\|_{\infty} \leq \frac{\|\gamma\|_{2}^{2}}{K^{2} C_{1}}
$$

LEMMA 3.3. For every $m>3 / 2$, the numbers $\alpha$ and $\beta$ can be found satisfying the inequality $\alpha>\beta>2$ such that $\|A\|<1$ for all $u_{0}$ small enough.

Proof. By the definition,

$$
\begin{aligned}
\|A\| & =\sup _{\|s\|_{\infty}=1}\|A s\|_{\infty} \leq \sup _{\|s\|_{\infty}=1} \sup _{n} \sum_{k=1}^{+\infty}\left|A_{n k} s_{k}\right| \\
& \leq \sup _{n} \sum_{k}\left|A_{n k}\right|=\sup _{n} \sum_{k \neq n} \frac{d_{n k}}{d_{n n}} \\
& =\sup _{n}\left(\sum_{k=1}^{n-1} \frac{d_{n k}}{d_{n n}}+\sum_{k=n+1}^{+\infty} \frac{d_{n k}}{d_{n n}}\right) .
\end{aligned}
$$

Hence, it suffices to require that

$$
S_{1}:=\frac{\left(\sum_{k=1}^{n-1} d_{n k}\right)}{d_{n n}}<\frac{1}{4}, \quad S_{2}:=\frac{\left(\sum_{k=n+1}^{+\infty} d_{n k}\right)}{d_{n n}}<\frac{1}{4} .
$$


Using (3.1), (3.2), (3.3), and (3.10), we get

$$
\begin{aligned}
& S_{1} \leq \frac{C_{2}}{C_{1}} \cdot \frac{\varepsilon_{n}^{m} u_{n}^{m-3}}{u_{n-1}^{2 m}}=\frac{C_{2}}{C_{1}} \cdot u_{n-1}^{\alpha(m-3)+(\beta-2) m}, \\
& S_{2} \leq \frac{C_{3}}{C_{1}} \cdot \frac{u_{n}^{m-3} u_{n+1}^{3}}{\varepsilon_{n+1}^{m}}=\frac{C_{3}}{C_{1}} \cdot u_{n}^{3 \alpha-m \beta+m-3} .
\end{aligned}
$$

Thus, if there exist $\alpha>\beta>2$ such that

$$
\alpha(m-3)+(\beta-2) m>0, \quad 3 \alpha-m \beta+m-3>0,
$$

then

$$
S_{1} \leq\left(\frac{C_{2}}{C_{1}}\right) \cdot u_{0}^{\alpha(m-3)+(\beta-2) m}, \quad S_{2} \leq\left(\frac{C_{3}}{C_{1}}\right) \cdot u_{0}^{3 \alpha-m \beta+m-3} .
$$

Therefore, (3.17) will be true for all $u_{0}$ small enough.

Let $m \geq 3$. The first inequality in (3.19) holds for all $\alpha>\beta>2$. The second one is true for $\alpha>(\beta m+3-m) / 3$. It follows that we can take any $\beta>2$ and $\alpha>\max \{\beta,(\beta m+3-m) / 3\}=(\beta m+3-m) / 3$. Note that this choice of $\alpha$ and $\beta$ is consistent with the choice made for $m \geq 3$ in Lemma 3.2.

Now let $m \in(3 / 2 ; 3)$. Then, (3.19) is equivalent to

$$
\alpha<\frac{(\beta m-2 m)}{(3-m)}, \quad \alpha>\frac{(\beta m+3-m)}{3} .
$$

As it must be $\alpha>\beta$, find $\beta$ such that

$$
\frac{(\beta m+3-m)}{3}<\beta<\frac{(\beta m-2 m)}{(3-m)} .
$$

The inequality $(\beta m+3-m) / 3<\beta$ is fulfilled for all $\beta>1$. The inequality $\beta<(\beta m-2 m) /(3-m)$ is equivalent to $\beta>2 m /(2 m-3)$. Hence, for $m \in$ $(3 / 2 ; 3)$, we can take any $\beta>\max \{2 ; 2 m /(2 m-3)\}=2 m /(2 m-3)$ and $\alpha \in$ $(\beta ;(\beta m-2 m) /(3-m))$. We also see that $\alpha$ and $\beta$ satisfy (3.13). This completes the proof.

By virtue of the inequality $\|A\|<1$, equation (3.9) has a unique solution in $l_{\infty}$

$$
\vec{c}=(I+A)^{-1} f .
$$


It is easily seen that all the components $c^{n}$ of the vector $\vec{c}=\left(c^{1}, c^{2}, \ldots\right)^{T}$ are nonnegative. Indeed, $f_{n}=\gamma_{n} / d_{n n}>0$ since, by (2.17) and (2.18), $\gamma_{n}, d_{n n}>$ 0 . The Neumann series $\vec{c}=\left(I+(-A)+(-A)^{2}+\cdots\right) f$ includes powers of a matrix with nonnegative entries such that $(-A)_{n k}=\left(1-\delta_{n k}\right) \cdot d_{n k} / d_{n n} \geq 0$ since $d_{n k} \geq 0$. Consequently, the vector $\vec{c}$ is obtained as a result of the action of a matrix with nonnegative entries on a vector whose components are also nonnegative.

4. Smoothness of the function $\varphi$. Introducing the convenient notations

$$
\begin{gathered}
u_{n}^{1}:=u_{n}, \quad u_{n}^{2}:=\frac{u_{n}}{2}, \quad \Delta_{n}:=\left[u_{n}^{2} ; u_{n}^{1}\right], \\
\Delta_{n}^{2}:=\left[u_{n}^{2} ; \frac{\left(u_{n}^{1}+u_{n}^{2}\right)}{2}\right], \quad \Delta_{n}^{1}:=\left[\frac{\left(u_{n}^{1}+u_{n}^{2}\right)}{2} ; u_{n}^{1}\right],
\end{gathered}
$$

we can write (2.11) as follows:

$$
\varphi_{n}(t):= \begin{cases}\omega\left(t-u_{n}^{2}\right), & t \in \Delta_{n}^{2}, \\ \omega\left(u_{n}^{1}-t\right), & t \in \Delta_{n}^{1}, \\ 0, & t \notin \Delta_{n} .\end{cases}
$$

LEMMA 4.1. Suppose that $\omega$ is monotone and semiadditive (and thus nonnegative). Then, the function

$$
\psi(t):=\sum_{k=1}^{+\infty}\left(c^{k}\right)^{1 / 2} \varphi_{k}(t)
$$

satisfies the following smoothness condition:

$$
|\psi(t+h)-\psi(t)| \leq \sup _{k}\left(c^{k}\right)^{1 / 2} \omega(|h|), \quad t, h \in \mathbb{R} .
$$

Inequality (4.4) remains true if $\left\{\Delta_{n}\right\}_{n=1}^{+\infty}$ is an arbitrary sequence of disjoint finite intervals.

Proof. If $t_{1} \in \Delta_{n}$ and $t_{2} \notin \Delta_{n}$, then, as the intervals $\Delta_{k}$ are disjoint for different $k$, for some $i \in\{1,2\}$, we have

$$
\psi\left(t_{1}\right)=\left(c^{n}\right)^{1 / 2} \cdot \omega\left(\left|t_{1}-u_{n}^{i}\right|\right) \leq\left(c^{n}\right)^{1 / 2} \cdot \omega\left(\left|t_{1}-t_{2}\right|\right) .
$$

Thus,

$$
\left|\psi\left(t_{2}\right)-\psi\left(t_{1}\right)\right| \leq \max \left\{\psi\left(t_{2}\right), \psi\left(t_{1}\right)\right\} \leq \sup _{k}\left(c^{k}\right)^{1 / 2} \cdot \omega\left(\left|t_{1}-t_{2}\right|\right) .
$$


For $t, \delta>0, \omega(t+\delta)-\omega(t) \leq \omega(\delta)$ according to the semiadditivity of $\omega$. Therefore, for $t_{1}, t_{2} \in \Delta_{n}^{i}$, we obtain

$$
\begin{aligned}
\left|\psi\left(t_{1}\right)-\psi\left(t_{2}\right)\right| & =\left(c^{n}\right)^{1 / 2} \cdot\left|\omega\left(\left|t_{1}-u_{n}^{i}\right|\right)-\omega\left(\left|t_{2}-u_{n}^{i}\right|\right)\right| \\
& \leq\left(c^{n}\right)^{1 / 2} \cdot \omega\left(\left|t_{1}-t_{2}\right|\right) .
\end{aligned}
$$

Now, let $t_{1} \in \Delta_{n}^{i}$ and $t_{2} \in \Delta_{n}^{j}, i \neq j$. Assume that $\left|t_{1}-u_{n}^{i}\right| \geq\left|t_{2}-u_{n}^{j}\right|$, then

$$
\begin{aligned}
\left|\psi\left(t_{1}\right)-\psi\left(t_{2}\right)\right| & =\left(c^{n}\right)^{1 / 2} \cdot\left(\omega\left(\left|t_{1}-u_{n}^{i}\right|\right)-\omega\left(\left|t_{2}-u_{n}^{j}\right|\right)\right) \\
& \leq\left(c^{n}\right)^{1 / 2} \cdot\left(\omega\left(\left|t_{1}-u_{n}^{j}\right|\right)-\omega\left(\left|t_{2}-u_{n}^{j}\right|\right)\right) \\
& \leq\left(c^{n}\right)^{1 / 2} \cdot \omega\left(\left|t_{1}-t_{2}\right|\right) .
\end{aligned}
$$

The lemma is proved.

By (2.12), the function $\varphi(t)=K \psi(t)+\gamma(t)$ where the function $\gamma$ is assumed to satisfy a Lipschitz condition $|\gamma(t+h)-\gamma(t)| \leq C_{\gamma}|h|, h \in \mathbb{R}$. Since the functions $\psi$ and $\gamma$ have disjoint supports and $\omega(t)=C_{\omega} t$, we see that the following inequality:

$$
\begin{aligned}
|\varphi(t+h)-\varphi(t)| & \leq K \sup _{k}\left(c^{k}\right)^{1 / 2} \cdot \omega(|h|)+C_{\gamma}|h| \\
& \leq\left(K \sup _{k}\left(c^{k}\right)^{1 / 2}+\frac{C_{\gamma}}{C_{\omega}}\right) \omega(|h|)
\end{aligned}
$$

holds for all $t, h \in \mathbb{R}$.

After finding the solution of system (2.14), we satisfy (2.13) replacing the function $\varphi$ by $\varphi / \sqrt{\left|\alpha_{m}\right|}$. This replacement corresponds to a passage from the functions $\psi$ and $\gamma$ to $\psi / \sqrt{\left|\alpha_{m}\right|}$ and $\gamma / \sqrt{\left|\alpha_{m}\right|}$, respectively. Therefore, for the new function $\varphi$, the following smoothness condition will be fulfilled:

$$
|\varphi(t+h)-\varphi(t)| \leq\left(K \sup _{k}\left(c^{k}\right)^{1 / 2}\left|\alpha_{m}\right|^{-1 / 2}+\frac{C_{\gamma}\left|\alpha_{m}\right|^{-1 / 2}}{C_{\omega}}\right) \omega(|h|), \quad h \in \mathbb{R} .
$$

LEMMA 4.2. The constant

$$
M:=K \sup _{k}\left(c^{k}\right)^{1 / 2}\left|\alpha_{m}\right|^{-1 / 2}+\frac{C_{\gamma}\left|\alpha_{m}\right|^{-1 / 2}}{C_{\omega}}
$$

in the smoothness condition (4.10), satisfies the inequality $M \leq C / K$, and hence, it is less than one for all $K$ large enough. 
Proof. Since

$$
\begin{aligned}
\left|\alpha_{m}\right| & =\int_{-\infty}^{+\infty} \frac{\varphi^{2}(t)}{\left.|\operatorname{sgn} t \cdot| t\right|^{m}-\lambda_{1} \mid} d t \\
& \geq K^{2} \int_{0}^{1} \frac{\psi^{2}(t)}{\lambda_{1}-t^{m}} d t \geq K^{2} \int_{0}^{1} \psi^{2}(t) d t \equiv K^{2}\|\psi\|_{2}^{2},
\end{aligned}
$$

it follows that $\left|\alpha_{m}\right|^{-1 / 2} \leq 1 /\left(K\|\psi\|_{2}\right)$. From (3.23), we obtain the inequality

$$
\|\vec{c}\|_{\infty} \leq(1-\|A\|)^{-1}\|f\|_{\infty} .
$$

In view of relation (3.15), then

$$
\|\vec{c}\|_{\infty} \leq(1-\|A\|)^{-1} \frac{\|\gamma\|_{2}^{2}}{K^{2} C_{1}} .
$$

Therefore, for the first summand in (4.11), we get

$$
K \sup _{k}\left(c^{k}\right)^{1 / 2}\left|\alpha_{m}\right|^{-1 / 2} \leq K(1-\|A\|)^{-1 / 2} \frac{\|\gamma\|_{2}}{K C_{1}^{1 / 2}} \cdot \frac{1}{K\|\psi\|_{2}} \leq \frac{C}{K} .
$$

Likewise, we find that

$$
\frac{C_{\gamma}\left|\alpha_{m}\right|^{-1 / 2}}{C_{\omega}} \leq \frac{C_{\gamma}}{C_{\omega}} \cdot \frac{1}{K\|\psi\|_{2}} \leq \frac{C}{K}
$$

The lemma is proved.

As a result, the function $\varphi$ satisfies the smoothness condition

$$
|\varphi(t+h)-\varphi(t)| \leq \omega(|h|), \quad h \in \mathbb{R},
$$

for all $K$ large enough. Theorem 1.1 is, thus, completely proved.

AcKnowledgment. The author expresses a deep gratitude to S. N. Naboko for his attention to this work and for useful remarks.

\section{REFERENCES}

[1] E. M. Dyn'kin, S. N. Naboko, and S. I. Yakovlev, A finiteness bound for the singular spectrum in a selfadjoint Friedrichs model, Algebra i Analiz 3 (1991), no. 2, 77-90.

[2] L. D. Faddeev, On a model of Friedrichs in the theory of perturbations of the continuous spectrum, Trudy Mat. Inst. Steklov 73 (1964), 292-313.

[3] S. I. Iakovlev, Perturbations of a singular spectrum in a selfadjoint Friedrichs model, Vestnik Leningrad. Univ. Mat. Mekh. Astronom. (1990), no. 1, 116117.

[4] Ya. V. Mikityuk, The singular spectrum of selfadjoint operators, Dokl. Akad. Nauk SSSR 303 (1988), no. 1, 33-36.

[5] S. N. Naboko, Uniqueness theorems for operator-valued functions with positive imaginary part and the singular spectrum in the selfadjoint Friedrichs model, Dokl. Akad. Nauk SSSR 275 (1984), no. 6, 1310-1313. 
[6] __ Uniqueness theorems for operator-valued functions with positive imaginary part, and the singular spectrum in the selfadjoint Friedrichs model, Ark. Mat. 25 (1987), no. 1, 115-140.

[7] S. N. Naboko and S. I. Yakovlev, Conditions for the finiteness of the singular spectrum in a selfadjoint Friedrichs model, Funktsional. Anal. i Prilozhen. 24 (1990), no. 4, 88-89.

[8] B. S. Pavlov and S. V. Petras, The singular spectrum of a weakly perturbed multiplication operator, Funkcional. Anal. i Priložen. 4 (1970), no. 2, 54-61.

[9] S. I. Yakovlev, On the structure of the singular spectrum in selfadjoint Friedrichs model, 1991, Depon. VINITI, pp. 1-25.

[10] _ A finiteness bound for the singular spectrum in a neighborhood of a singular point of operators of the Friedrichs model, Algebra i Analiz 10 (1998), no. $4,210-237$.

Serguei I. Iakovlev: Departamento de Matemáticas, Universidad Simón Bolívar, Apartado Postal 89000, Caracas 1080-A, Venezuela

E-mail address: serguei@usb.ve 


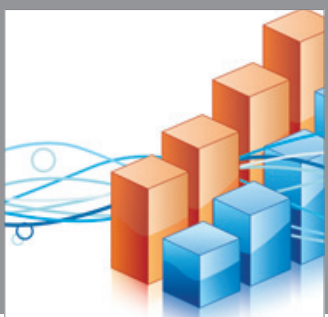

Advances in

Operations Research

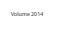

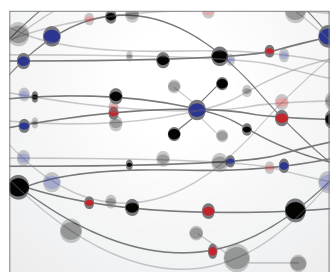

\section{The Scientific} World Journal
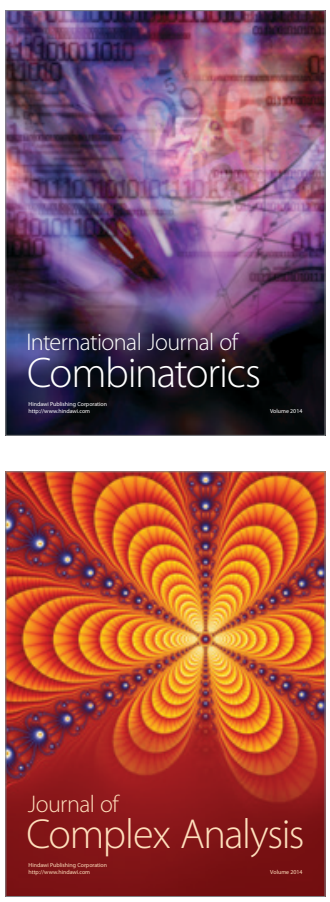

International Journal of

Mathematics and

Mathematical

Sciences
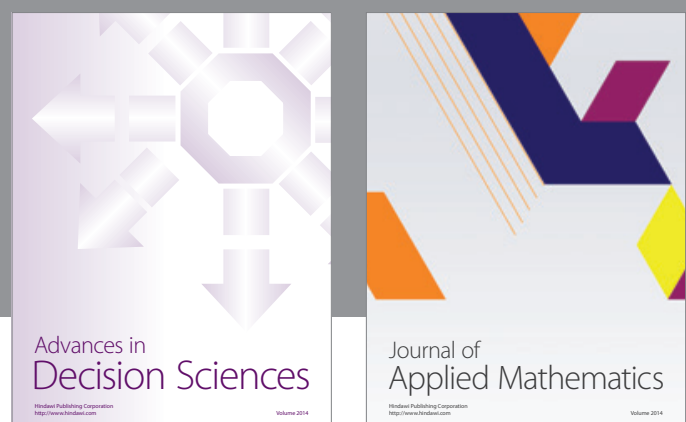

Journal of

Applied Mathematics
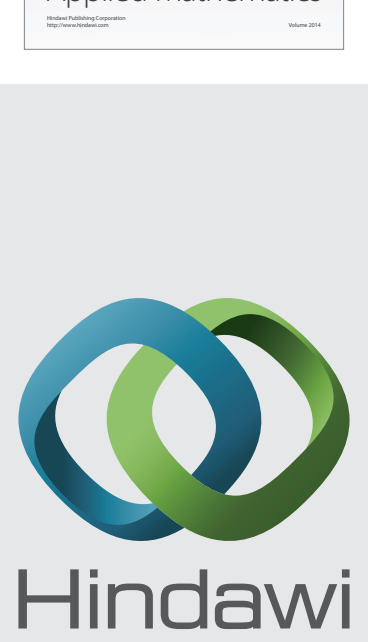

Submit your manuscripts at http://www.hindawi.com
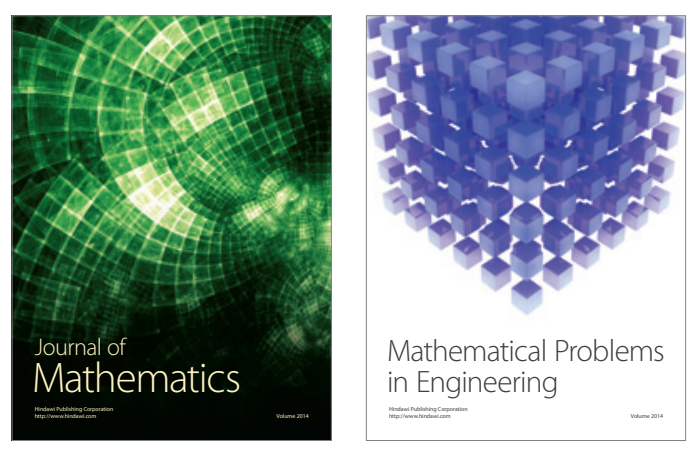

Mathematical Problems in Engineering
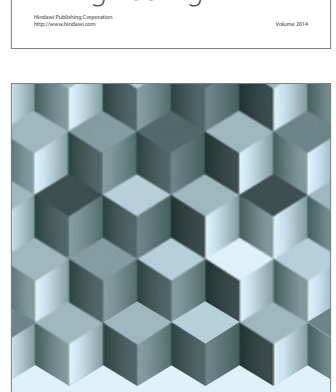

Journal of

Function Spaces
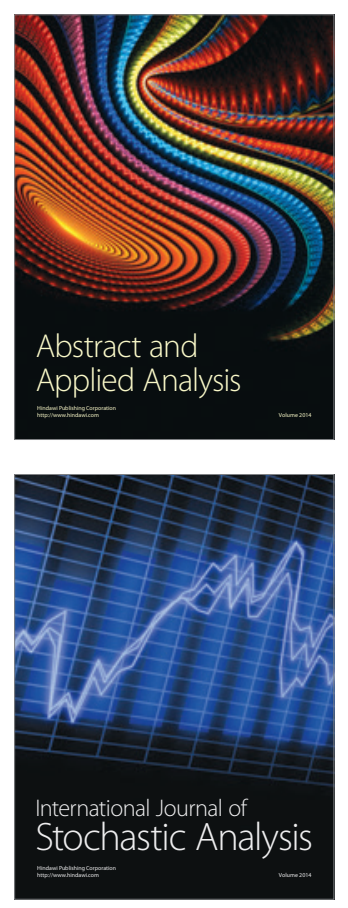

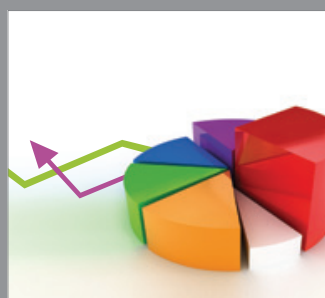

ournal of

Probability and Statistics

Promensencen
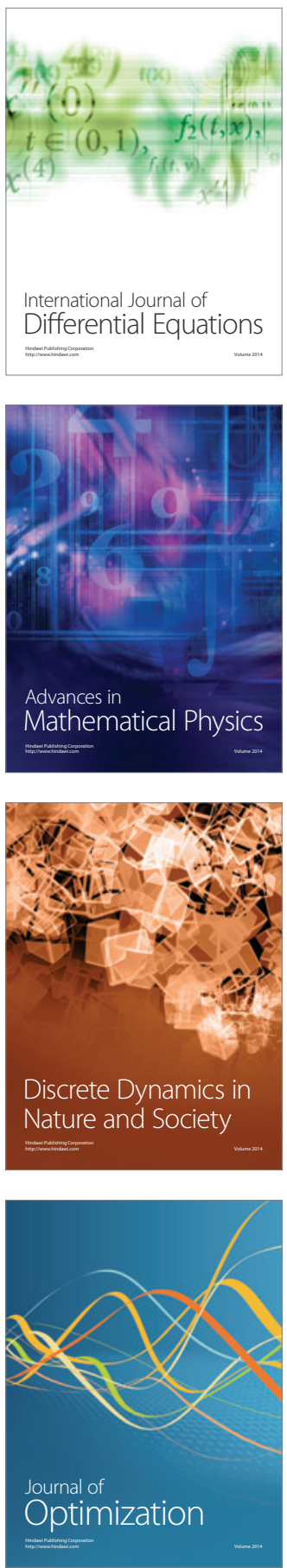